\title{
LOS FITOESTRÓGENOS Y EL EFECTO DE SU CONSUMO EN DIFERENTES ÓRGANOS Y SISTEMAS DE ANIMALES DOMÉSTICOS
}

\author{
Phytoestrogens consumption and their effects in different organs and systems \\ of domestic animals
}

\author{
Juan Pérez-Rivero ${ }^{1}$, Álvaro Aguilar-Setién ${ }^{2}$, José Martínez-Maya ${ }^{1}$, \\ Mario Pérez-Martínez ${ }^{1}$ y Héctor Serrano ${ }^{3}$ *
}

\begin{abstract}
A B S T R A C T
Phytoestrogens are plant-derived compounds having similar effects to estrogens. Several phytostrogen groups have been identified being the most characteristics the isoflavones like genistein, coumestans like coumestrol, and lignans represented by the enterolactone. Depending on the doses, they can act as agonists or antagonists of steroidal hormones. This apparently bimodal function is due to their capacity to interact with the alpha form of the estrogen receptor $(E R \alpha)$ in several organs of the female reproductive tract and to the beta form ER $\beta$ present in prostate, testis, ovaries, limph nodes and brain regions. It has been observed that an increase in genistein or coumestrol induces an increase in haemorrhagic follicles, miscarriages, LH-FSH surge suppression and even estrogenic syndrome, whereas in males, induces alterations in testis development and a decrease in sperm count. In this review, a panoramic view of the actual research of phytostrogen impact on the reproductive function and perspectives mainly for domestic animals is presented.
\end{abstract}

Key words: coumestrol, genistein, estrogen receptors.

\section{R E S U M E N}

Los fitoestrógenos son substancias de origen vegetal con propiedades similares a los estrógenos. Se han identificado diferentes grupos de fitoestrógenos entre los que destacan las isoflavonas como la genisteina, los coumestanos como el coumestrol, y los lignanos representados por la enterolactona. Estos compuestos actúan como agonistas o como antagonistas de las hormonas esteroidales dependiendo de la dosis que se utilice. Esta acción aparentemente contradictoria se debe a su capacidad para unirse como ligandos a los receptores estrogénicos alfa $(\mathrm{ER} \alpha)$ del útero, glándula mamaria, sistema cardiovascular y hueso, y con mayor afinidad a los receptores estrogénicos beta (ER $\beta)$ presentes en próstata, ovarios, testículos, tracto urinario, tejido linfoide y algunas regiones del cerebro como el hipotálamo. Se ha observado que a medida que se incrementa la dosis de genisteína y coumestrol, se inducen folículos hemorrágicos, abortos, síndrome estrogénico, y supresión de picos de hormona luteinizante (LH) en las hembras, mientras que en los machos se altera el desarrollo testicular y disminuye el recuento de espermatozoides. En esta revisión se presenta un panorama del estado actual de las investigaciones respecto a las implicaciones de los fitoestrógenos sobre la reproducción y sus perspectivas de estudio, poniendo énfasis en su importancia en los animales domésticos.

Palabras clave: coumestrol, genisteína, receptores estrogénicos

\footnotetext{
${ }^{1}$ Universidad Nacional Autónoma de México, Facultad de Medicina Veterinaria y Zootecnia, México, DF.

${ }^{2}$ Centro Médico Nacional Siglo XXI, Unidad de Investigación en Inmunología, México, DF.

${ }^{3}$ Universidad Autónoma Metropolitana - Iztapalapa, Departamento de Ciencias de la Salud, San Rafael Atlixco 186, México, 09340, DF, México. E-mail: hser@xanum.uam.mx *Autor para correspondencia.

Recibido: 3 de marzo de 2006. Aceptado: 28 de junio de 2006.
} 


\section{INTRODUCCIÓN}

Una de las metas en el manejo del ganado vacuno u ovino es generar, dentro de los márgenes posibles, el máximo rendimiento. Ocasionalmente, pueden presentarse problemas reproductivos cuya causa es difícil detectar. Una posibilidad es que no se trate realmente de un problema asociado al manejo reproductivo u orgánico, sino que sea sólo el reflejo de la presencia de compuestos que simulan el efecto de las hormonas esteroides y que involuntariamente se administran al ganado en el alimento. En esta revisión queremos hacer notar el papel que juegan los fitoestrógenos en este problema y la posible repercusión económica que implicaría su efecto sobre la reproducción.

Los fitoestrógenos son productos de origen vegetal con actividad natural bactericida y fungicida de patógenos vegetales (Pike et al., 1999). Estos compuestos presentan una actividad similar a los estrógenos, a pesar de que la estructura química es bastante diferente (Figura 1). Existen tres grupos de estos compuestos: las isoflavonas, los coumestanos, y los lignanos, cuyos principales representantes son la genisteína (GEN), el coumestrol (COU) y la enterolactona, respectivamente (Whitten y Patisaul, 2001; Whitten et al., 2002).

Una planta puede tener más de una clase de fitoestrógenos, por ejemplo, las semillas de soya ( $\mathrm{Glyci}$ ne sp.), son ricas en isoflavonas, mientras que el

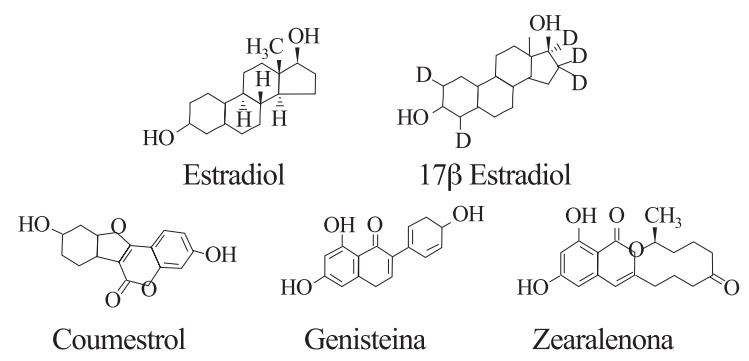

Figura 1. Estructura de diferentes compuestos con actividad estrogénica. Estructuras disponibles en http://www.sigmaaldrich.com/catalog/search/ ProductDetail/

Figure 1. Chemical structure of different estrogenic activity compounds. Structure drawings available at http://www.sigmaaldrich.com/catalog/search/ ProductDetail/ germen de soya es la principal fuente del coumestano (Murkies et al., 1998). Asimismo, el COU también se encuentra en la alfalfa (Medicago sativa L.) (Jacob et al., 2001), y su concentración aumenta cuando se encuentra contaminada por el hongo Pseudopeziza medicaginis (Romero et al., 1997). En las plantas, las isoflavonas GEN y daidzeina (DEZ) están presentes como glucósidos inactivos (Rosselli et al., 2000).

La importancia de los fitoestrógenos radica principalmente en que pueden actuar como agonista o antagonista estrogénico (Whitten et al., 2002), ya que al igual que los estrógenos, ejercen su influencia en tejidos donde existen receptores para estrógenos (ER), tanto en el macho como en la hembra (Rosselli et al., 2000).

Como se sabe, los estrógenos favorecen la diferenciación celular y el crecimiento de las glándulas mamarias, útero, vagina, ovarios, testículos, epidídimo, próstata (Rosselli et al., 2000), sistema vascular (Dubey et al., 2000) y sistema nervioso (McGarvey et al., 2001).

Las isoflavonas también se unen a los receptores para andrógenos (AR), no estando claro hasta el momento cuál es el mecanismo del efecto antiandrógeno de estos compuestos (Beck et al., 2005).

Se ha observado en ratas que el consumo de 40 a $50 \mathrm{mg}$ de GEN, equivalentes a los niveles séricos encontrados en humanos que consumen dietas ricas en isoflavonas, diminuye el número de los AR y los ER en la próstata. Así mismo, algunos fitoestrógenos como las isoflavonas se unen en forma débil a receptores para progesterona (PR) (Beck et al., 2005).

Hasta el momento no se conoce plenamente el metabolismo de los fitoestrógenos. Respecto al COU, Yamada et al. (1999) proponen que este compuesto sufre modificaciones en su estructura, que van desde hidroxilaciones, desmetilaciones e incluso hidrólisis, por lo que se forman más de diez intermediarios, de los cuales algunos son detectados en la orina. Estas modificaciones incluyen algunas etapas donde intervienen bacterias residentes del tracto digestivo, procesos de absorción y reabsorción en el hígado, transporte en arterias y venas para su acumulación y desecho en la orina (Murkies et al., 1998; Rosselli et al., 2000). 


\section{Receptores estrogénicos}

Como miembros de la superfamilia de receptores nucleares, los receptores estrogénicos (ER) tienen estructura modular (Figura 2). Se han identificado las isoformas $\alpha(E R \alpha)$ y $\beta(E R \beta)$, que se distinguen por presentar diferencias en su secuencia de aminoácidos. Estos receptores presentan 96\% de homología de sus aminoácidos constituyentes, aunque no es una regla aplicable a todas las regiones del receptor, ya que la región de unión al ADN (DBD) es la que más similitud presenta, mientras que la región de unión al ligando (LBD) sólo presenta un $53 \%$ de similitud (Weihua et al., 2003).

$\mathrm{ER} \alpha$

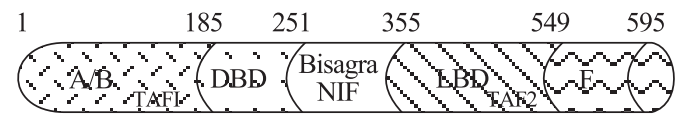

$\operatorname{ER} \beta$

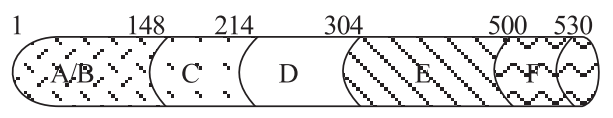

Figura 2. Estructura modular de los receptores estrogénicos (ER). Se representa la distribución que abarcan cada uno de los dominios de los receptores para estrógenos alfa (ER $\alpha)$ y beta (ER $\beta)$, como lo indican los números de residuos de aminoácidos colocados encima de cada uno. La región de unión al ADN (DBD) es la que se conserva más entre ambos receptores; la bisagra (D) contiene la secuencia de localización nuclear (NIF), el cual guía al receptor desde el citoplasma hacia el núcleo. La región de unión al ligando (LBD) es la menos conservada entre los dos receptores, este receptor tiene la región (F), caso único entre los receptores nucleares, la cual se encarga de diferenciar el efecto de agonista $o$ antagonista cuando se une el ligando (Modificado de Pérez-Rivero et al., 2005).

Figure 2. Modular structure of estrogen receptors (ER). The distribution of each domain of the alpha $(E R \alpha)$ and betta $(E R \beta)$ receptor type as indicated by the aminoacid residue number above the diagram, is shown. The DNA binding region (DBD) is the most conserved domain in $\alpha$ and $\beta$ receptors; nuclear localization sequences are located in the hinge region (D) allowing the receptor travel to the nucleus. The less conserved region between the two ER types is the ligand binding domain (LBD). The $F$ region is the most characteristic domain for ER and is absent in most nuclear receptors. The $F$ domain is responsible for the agonist or antagonist effect induced by the bound ligand (Modified from Pérez-Rivero et al., 2005).
Los estrógenos presentes en el plasma penetran a sus células blanco en los tejidos llamados "clásicos", que se encuentran en el útero, glándula mamaria, placenta, hígado, sistema nervioso central, sistema cardiovascular y hueso. Las células blanco de estos tejidos presentan abundantes ER $\alpha$ (Mosselman et al., 1996; Weihua et al., 2003). Existen otros tejidos considerados "no clásicos", en los que la presencia de ER $\alpha$ es relativamente menor, pero que contienen cantidades significativamente mayores de ER $\beta$. Entre estos últimos se incluyen próstata, ovarios, testículos, glándula pineal, glándula tiroides, paratiroides, suprarrenales y páncreas, vesícula biliar, piel, tracto urinario, tejido linfoide, tejido eritroide, pulmón, epitelio intestinal, y algunas regiones del cerebro, como el hipotálamo, cerebelo y lóbulo olfatorio (Jacob et al., 2001). El tejido muscular estriado es único, puesto que en él se expresa de manera elevada el ER $\beta$ y está casi ausente el ER $\alpha$ (Mosselman et al., 1996; Weihua et al., 2003).

En células de la línea celular identificada en la American Type Cell Cultural Collection como T47D originada a partir de un tumor mamario, así como en diferentes tejidos como el prostático y el epitelio de la glándula mamaria, los estrógenos actúan en presencia del ER $\alpha$ como agonista, favoreciendo la proliferación de los tejidos. El ER $\beta$ actúa de manera antagonista, inhibiendo la proliferación del tejido, por lo que una misma hormona produce efectos opuestos, dependiendo del receptor al que se una (Imamov et al., 2005).

Los ER son receptores nucleares que modulan la trascripción por su unión específica a secuencias del genoma, así como a co-represores y co-activadores, para regular la acción del complejo de la ARN polimerasa. Los efectos moduladores de los ER sobre la trascripción se llevan a cabo en una serie secuencial de eventos. La unión de los ER con los estrógenos induce cambios en la conformación del receptor, que le permite al mismo tiempo disociarse de un complejo co-represor y unirse con un complejo co-activador, lo cual le confiere la actividad de trascripción de genes que contienen elementos de respuesta a los estrógenos (ERE). Por el contrario, en ausencia de estrógenos, los ER se asocian con los co-represores que inhiben la actividad de trascripción (Maniatis y Reed, 2002; Hewitt y Korach, 2003). 
Se sabe que el coumestrol (3,9-dihidroxi-6h-benzofuro[3,2-c][1]benzo-piran-6-ona) se une al ER $\beta$ con una afinidad similar a la del $17 \beta$ estradiol (Morito et al., 2002), pero tres veces menos que el estradiol para el ER $\alpha$ (Kuiper et al., 1998). La inducción de la trascripción es similar entre el COU y la GEN, uniéndose el coumestrol con mayor afinidad al ER $\alpha$ que la GEN, la cual lo hace preferentemente al ER $\beta$ (Morito et al., 2002).

Las isoflavonas tienen poca afinidad por la hormona transportadora de esteroides (SHBG), ya que se han detectado fracciones libres que varían entre el 45 al $50 \%$ tanto de COU como de GEN en suero humano, mientras que solamente se ha encontrado el 4\% de estrógenos libres (Whitten y Patisaul, 2001).

\section{ACCIÓN DE LOS FITOESTRÓGENOS EN DIFERENTES ÓRGANOS Y SISTEMAS}

En el cerebro el ER $\beta$ se expresa principalmente en el área preóptica, en el núcleo ventro-medial del hipotálamo y en el tallo cerebral. Estudios realizados en ratones hembras utilizando COU indican que éste antagoniza la acción del estradiol mediante la regulación del mARN para el ER $\beta$ en el núcleo paraventricular (Jacob et al., 2001).

De manera similar, las isoflavonas GEN y DEZ tienen efectos en el núcleo paraventricular y ventromedial, áreas que regulan el comportamiento sexual y la secreción de gonadotropinas, induciendo cambios en los receptores para PR y en los ER $\beta$ (Whitten et al., 2002).

Se ha atribuido principalmente al fitoestrógeno COU la capacidad de suprimir los picos de la hormona luteinizante (LH) en ratas (McGarvey et al., 2001), y en ovejas (Jacob et al., 2001). La presencia de los ER $\alpha$ y ER $\beta$ en las neuronas secretoras de la hormona liberadora de gonadotropinas $(\mathrm{GnRH})$ abre la posibilidad que los fitoestrógenos actúen directamente sobre ellas (McGarvey et al., 2001).

El sistema vascular expresa el ER en su forma $\alpha$ y $\beta$, al que los fitoestrógenos se unen con diferente afinidad, y ejercen su efecto biológico tanto en las células endoteliales como en las del músculo liso. Al respecto, se ha informado que la GEN inhibe la proliferación de las células endoteliales siendo capaz de inhibir las señales de transducción que están involucradas en trastornos vaso-oclusivos así como en los procesos de carcinogénesis/angiogénesis ejerciendo un efecto vaso protector (Dubey et al., 2000).
Las isoflavonas tienen un efecto bifásico que favorece la formación de hueso cuando se encuentran en bajas concentraciones o la reabsorción del mismo cuando están presentes a altas concentraciones. Esto se ha demostrado en el modelo de roedores ovariectomizados a los que se les administraron bajas dosis de GEN $\left(0,5 \mathrm{mg} \mathrm{d}^{-1}\right)$, lo que promueve la retención ósea, no así cuando se incrementa la dosis a $5 \mathrm{mg} \mathrm{d}^{-1}$, debido a que el tejido óseo es más sensible a las isoflavonas que el útero. El ER $\alpha$ está presente en el hueso y es el encargado de mediar los efectos estrogénicos en el mismo. Sin embargo, cuando existen concentraciones micromolares de fitoestrógenos, las isoflavonas se unen preferentemente a los ER $\beta$ cuyo efecto es una función reguladora negativa en el tejido óseo. Este fenómeno puede explicar en parte este efecto bifásico. Sin embargo, el efecto antiestrogénico de las isoflavonas también se debe a la competencia entre los fitoestrógenos y los estrógenos para unirse a los ER (Dang y Lowik, 2005).

\section{Los fitoestrógenos en el aparato reproductor}

Se ha tratado de aprovechar el efecto de los fitoestrógenos con resultados poco alentadores. La premisa en la cual se basan estos estudios es que a través del uso de los fitoestrógenos es posible aumentar la frecuencia de estros en el ganado vacuno, lo que podría tener un impacto positivo en la producción de productos cárnicos o lácteos. Sin embargo, los resultados son totalmente diferentes. Así, en vacas Holstein que consumen 25 a $100 \mathrm{mg} \mathrm{kg}^{-1} \mathrm{de}$ zearalenona por 42 días, muestran genitales externos edematosos, como si fuesen a presentar un estro fisiológico. Sin embargo, la duración del ciclo estral o el tiempo en el que se presenta permanecen inalterados (Mirocha et al., 1978).

En el otro extremo, se ha reportado que el COU causa alteraciones reproductivas en hembras de diferentes especies (Cuadro 1). Los ratones hembra prepúberes de la cepa Swiss Webster que han sido alimentados durante cinco días con $20 \mathrm{~g}$ de alimento que contenga intencionalmente $90 \mathrm{mg} \mathrm{kg}^{-1} \mathrm{de}$ coumestrol, presentaron edema uterino (Galey et al., 1993). Por su parte, la administración de 100 $\mu \mathrm{g}$ de $\mathrm{COU}$ en ratones hembra de 1 a 5 días de nacidos produce cambios en los ovarios como es la presencia de folículos hemorrágicos y folículos poliovulares (Burroughs, 1995). En ratas SpragueDawley se encontró que el COU se une con afinidad similar al estradiol a los ER presentes en el útero (Branham et al., 2000). 
Cuadro 1. Efecto de los fitoestrógenos en la fisiología reproductiva de especies experimentales y de importancia zootécnica.

Table 1. Phytoestrogen effects on reproductive physiology in experimental and zootechnical important species.

\begin{tabular}{|c|c|c|c|c|c|c|}
\hline Animal & Edad & Sexo & Fitoestrógeno & Dosis y vía & Efecto & Referencia \\
\hline Rata Wistar & 2 días & M & Coumestrol & $\begin{array}{l}4 \mathrm{mg} \mathrm{kg}^{-1} \mathrm{~d}^{-1} \\
\mathrm{SC} \text { por16 días }\end{array}$ & $\begin{array}{l}\text { Disminución de lumen de } \\
\text { túbulos seminíferos, incre- } \\
\text { mento del índice apoptótico } \\
\text { en células germinales }\end{array}$ & $\begin{array}{l}\text { Atanassova } \\
\text { et al., } 2000\end{array}$ \\
\hline \multirow[t]{2}{*}{ Rata Wistar } & Adultas & $\mathrm{H}$ & Coumestrol & $\begin{array}{l}0,4-1,6 \mathrm{mg} \text { IV } \\
\text { por } 8,5 \mathrm{~h}\end{array}$ & $\begin{array}{l}\text { Inhibición de la hormona } \\
\text { luteinizante }(\mathrm{LH}) \text { por inhibición } \\
\text { de Hormona Liberadora }\end{array}$ & $\begin{array}{l}\text { McGarvey, } \\
\text { et al., } 2001\end{array}$ \\
\hline & & $\mathrm{H}$ & Genisteina & $\begin{array}{l}1,6 \mathrm{mg} \mathrm{IV} \\
\text { por } 8,5 \mathrm{~h}\end{array}$ & $\begin{array}{l}\text { de Gonadotropinas } \\
(\mathrm{GnRH})\end{array}$ & \\
\hline \multirow{4}{*}{$\begin{array}{l}\text { Rata } \\
\text { Sparague } \\
\text { Dawley }\end{array}$} & Adultas & $\mathrm{H}$ & Coumestrol & $100 \mathrm{mg} \mathrm{kg}^{-1}$ & Anovulación, supresión & Shaw y \\
\hline & & & & en la dieta & de LH & McCully, \\
\hline & & & & & & 2002 \\
\hline & & M & & por 10 días & $\begin{array}{l}\text { Supresión de conducta sexual } \\
\text { como monta y eyaculación }\end{array}$ & \\
\hline Bovinos & Adultos & M & Coumestrol & $\begin{array}{l}66,8 \mathrm{mg} \mathrm{kg}^{-1} \\
\text { en alfalfa seca } \\
\text { como pastura } \\
\text { vía oral }\end{array}$ & $\begin{array}{l}\text { Metaplasia glandular } \\
\text { y epitelial en próstata } \\
\text { y glándulas bulbo-uretrales, } \\
\text { disminuye la maduración de } \\
\text { espermatozoides. }\end{array}$ & $\begin{array}{l}\text { Pike et al., } \\
1999\end{array}$ \\
\hline Ovejas & Adultos & $\mathrm{H}$ & Coumestrol & $\begin{array}{l}25 \mathrm{mg} \mathrm{kg}^{-1} \\
\text { oral en la } \\
\text { pastura }\end{array}$ & $\begin{array}{l}\text { Crecimiento uterino, descargas } \\
\text { vaginales, desarrollo mamario, } \\
\text { ovarios quísticos, estros irregulares }\end{array}$ & $\begin{array}{l}\text { Adams, } \\
1995\end{array}$ \\
\hline
\end{tabular}

Abreviaturas utilizadas: IV: intravenoso; H: hembras; M: machos; mg: miligramo; $\mu$ g: microgramo; SC: subcutáneo.

Los fitoestrógenos se encuentran presentes en diferentes plantas utilizadas como forraje para los rumiantes, como la alfalfa que contiene COU, trébol subterráneo (Trifolium subterraneum) que contiene las isoflavonas formononetina, biochamina A y GEN; el trébol rojo (Trifolium pratense) antes de la floración tiene un alto contenido de formononetina, que se mantiene cuando es ensilado o se convierte en forraje seco pero cuando el trébol rojo florece, la cantidad de isoflavonas disminuye (Adams, 1995).

Adams (1995) encontró una asociación entre la estrogenicidad en ovejas y el consumo de tréboles. Cuando la pastura con la cual se alimentaban las hembras contenía $25 \mathrm{mg} \mathrm{kg}^{-1}$ de COU, se presentó una baja tasa de nacimientos, folículos hemorrágicos, prolapso de útero, metritis y edema uterino. En las vacas lecheras alimentadas con alfalfa seca que tenía $68,8 \mathrm{mg} \mathrm{COU} \mathrm{kg}^{-1}$ de alfalfa, se presenta un síndrome estrogénico con características similares al descrito para ovejas (Romero et al., 1997).
Por otra parte, los fitoestrógenos también alteran la expresión de otros receptores nucleares importantes en la reproducción de la hembra, como los de oxitocina, los de testosterona además de los de PR (Whitten et al., 2002). Los fitoestrógenos pueden influenciar de manera negativa los ciclos foliculares en los ovarios de los diferentes mamíferos, ya que éstos interactúan con los ER $\beta$ presentes de manera abundante en las células de la granulosa, lo que disminuye el efecto de los estrógenos en los folículos (Whitten y Patisaul, 2001).

Los ER $\beta$ se expresan en diferentes regiones en el testículo, como los conductos eferentes, epidídimo, conductos deferentes y próstata (Saunders, 2005). Asimismo se sabe que existen en las células de Sertoli (Atanassova et al., 2000), espermatogonias y espermatocitos (Nie et al., 2002). En las células de Leydig de los adultos se expresan los ER $\alpha$ (Saunders, 2005).

In vitro, la GEN induce la apoptosis en líneas celulares derivadas de células de Leydig de ratón de manera dependiente de la concentración adminis- 
trada. Asimismo produce disminución en el número de montas y eyaculaciones en ratones (Iwase et al., 2006). En ratas macho de 2 días de nacidos a los cuales se les inyectan $4 \mathrm{mg} \mathrm{kg}^{-1}$ de GEN por vía subcutánea durante 16 días, se inhibe el desarrollo testicular a corto y largo plazo. Asimismo, cuando llegan a la edad adulta, se afecta el número de crías por camada (Atanassova et al., 2000).

En un estudio efectuado en toros, la ingestión de pasturas que contienen COU ocasiona metaplásia glandular y epitelial tanto en próstata como glándulas bulbo-uretrales. Asimismo, se presenta mayor número de espermatozoides inmaduros y con una disminución significativa en la movilidad (Romero et al., 1997).

El efecto del tratamiento con fitoestrógenos en el ganado bovino es relativamente escaso, sin embargo, se puede estimar. Así pues, en 608 vacas alimentadas con alfalfa contaminada con el hongo Pseudopeziza medicaginis, se obtuvo menos del $30 \%$ de gestaciones a pesar de haberse realizado 1264 inseminaciones en el lapso de un año. Este porcentaje de gestaciones se redujo en un $10 \%$ adicional al incorporar el número de vacas que tuvieron abortos espontáneos. Aún en las que presentaron gestaciones normales, los niveles de estrógenos estaban alterados, sintomatología característica del síndrome estrogénico (Romero et al., 1997). En cerdos, la ingesta de $25-50 \mathrm{mg} \mathrm{kg}^{-1}$ de zearalenona promueve una disminución en el número de lechones nacidos así como el tamaño de las crías (Sharma et al., 1994). Si a estos resultados se incorpora la pérdida económica y los gastos de manutención del ganado, es entendible la razón por la cual es difícil realizar este tipo de estudios.

El impacto de la ingestión de fitoestrógenos sobre la industria, puede ejemplificarse con lo que sucede en Australia. Rebaños de ovejas que fueron alimentados con pastura conteniendo trébol rojo vieron afectadas su capacidad reproductiva de tal forma, que cerca de un millón de ovejas no pudieron procrear, lo que disminuyó entre 5 a $8 \%$ las ganancias brutas al considerar las pérdidas en inversión, alimentación, cuidados, e incluso compra de ganado, además de la disminución en la producción de lana y la necesidad de comprar e importar animales (Adams, 1990).

\section{CONCLUSIONES}

Basado en la literatura consultada, se puede concluir que los fitoestrógenos pueden inducir alteraciones en el sistema endocrino de los animales que los consumen, o en los animales a los que se les administran con fines experimentales. Desde el punto de vista clínico, en bovinos y ovinos estos productos pueden generar pérdidas económicas considerables debido a las alteraciones reproductivas que ocasionan. Si bien en la literatura se tienen documentados los efectos que tienen los fitoestrógenos en animales de laboratorio y, aunque de manera escasa en animales de importancia económica, es preocupante para el productor y el médico encargado de la revisión del ganado, la detección de casos de alteración reproductiva cuya causa no pueda explicarse fácilmente. Una de las alternativas es a través de substancias con actividad estrogénica proveniente del alimento que, como hemos mencionado, puede estar asociado a alguno de los grupos de fitoestrógenos descritos en esta revisión.

La implementación de metodologías que permitan evaluar de manera simple, eficiente y de bajo costo la presencia de fitoestrógenos está aún en etapa de investigación. Si bien los fitoestrógenos pueden formar parte de los metabolitos secundarios o inducidos en los vegetales ante la presencia de patógenos, o como parte normal de su desarrollo, la evaluación al menos ocular de aspectos simples que estén asociados con la producción o acumulación de fitoestrógenos, como la floración del trébol rojo o la infestación de hongos en la alfalfa, permitirá un control que pueda reducir las pérdidas económicas ocasionadas por los fitoestrógenos. Aún falta mucho por estudiar respecto al impacto que tienen los fitoestrógenos en el desarrollo y función reproductiva de los animales así como la forma de antagonizar su efecto.

\section{LITERATURA CITADA}

Adams, N.R. 1990. Permanent infertility in ewes exposed to plant oestrogens. Aust. Vet. J. 67:197-201.

Adams, N.R. 1995. Detection of the effects of phytoestrogens on sheep and cattle. J. Anim. Sci. 73:1509-1515.
Atanassova, N., C. McKinnell, K. Turner, M. Walker, S. Fisher, M. Morley, et al. 2000. Comparative effects of neonatal exposure of male rats to potent and weak (environmental) estrogens on spermatogenesis at puberty and the relationship to adult testis size and fertility: evidence for stimulatory effects of low estrogen levels. Endocrinology 141:3898-3907. 
Branham, W.S., S.L. Dial, C.L. Moland, B.S. Hass, R.M. Blair, H. Fang, et al. 2000. Phytoestrogens and mycoestrogens bind to the rat uterine estrogen receptor. J. Nutr. 132:658-664.

Beck, V., U. Rohr, and A. Jungbauer. 2005. Phytoestrogens derived from red clover: an alternative to estrogen replacement therapy. J. Steroid Biochem. Mol. Biol. 94:499-518.

Burroughs, CD. 1995. Long-term reproductive tract alterations in female mice treated neonatally with coumestrol. Exp. Biol. Med. 208:78-81.

Dang, Z., and C. Lowik. 2005. Dose-dependent effects of phytoestrogens on bone. Trends. Endocrinol. Metabol. 16:207-213.

Dubey, K., R.M. Rosselli, J. Imthurn, P. Séller, and E.K. Jackson. 2000. Vascular effects of environmental oestrogens: implications for reproductive and vascular health. Hum. Reprod. Update 6:351-363.

Galey, D., E. Mendez, E. Whitehead, M. Holstege, H. Plimlee, and B. Johnson. 1993. Estrogenic activity in forages: diagnostic use of the classical mouse uterine bioassay. J. Vet. Diagn. Invest. 6:603-608.

Hewitt, S.C., and K.S. Korach. 2003. Oestrogen receptor knockout mice: roles for estrogen receptors $\alpha$ and $\beta$ in reproductive tissues. Reproduction 125:143-149.

Imamov, O., G. Shim, M. Warner, and J. Gustafsson. 2005. Estrogen receptor beta in health and disease. Biol. Reprod. 73:866-871.

Iwase, Y., H. Fukata, and C. Mori. 2006. Estrogenic compounds inhibit gap junctional intercellular communication in mouse Leydig TM3 cells. Toxicol. Appl. Pharmacol. 212:237-246.

Jacob, D.A., J.L. Temple, H.B. Patisaul, L.J. Young, and E.F. Rissman. 2001. Coumestrol antagonizes neuroendocrine actions of estrogen via the estrogen receptor a. Exp. Biol. Med. 226:301-306.

Kuiper, G.G., J.G. Lemmen, B. Carlsson, J.C. Corton, S.H. Safe, P.T. van der Sagg, et al. 1998. Interaction of estrogenic chemicals and phytoestrogens with estrogen receptor $\beta$. Endocrinology 139:4252-4263.

Maniatis, T., and R. Reed. 2002. An extensive network of coupling among gene expression machines. Nature (London) 416:499-506.

McGarvey, C., C. Cates, P.N. Brooks, A. Swanson, I.R. Milligan, W. Coen, et al. 2001. Phytoestrogens and gonadotropin-releasing hormone pulse generator activity and pituitary luteinizing hormone release in the rat. Endocrinology 142:1202-1208.

Mirocha, C.J., G.Weaver, B. Gustafsson, M. Chi, S.V. Pathre, T.S., Robinson, and F. Bates. 1978. Pharmacological and toxicological studies on zearalenone in food producing animals. $125 \mathrm{p}$. Quarterly Report 11. FDA, Washington DC, USA.

Morito, K., T. Aomori, T. Hirose, J. Kinjo, J. Hasegawa, S. Ogawa, et al. 2002. Interaction of phytoestrogens with estrogen receptor $\alpha$ and $\beta(\mathrm{II})$. Biol. Pharmaceut. Bull. 25:48-52.
Mosselman J., R. Polman, and R. Dijkema. 1996. ERß: identification and characterization of a novel human estrogen receptor. FEBS Lett. 392:49-53.

Murkies A.L., G. Wilcox, and R. Davis. 1998. Phytoestrogens. J. Clin. Endocrinol. Metabol. 83:297-303.

Nie, R., Q. Zhou, E. Jassmin, P.T. Saunders, and R.A. Hess. 2002. Differential expression of estrogen receptors $\alpha$ and $\beta$ in the reproductive tracts of adults male dogs and cats. Biol. Reprod. 66:1161-1168.

Pérez-Rivero, J.J., Aguilar-Setién, A., Villa-Godoy, A., y Serrano, H. 2005. Relación entre estructura y función de receptores para hormonas esferoidales: Receptores estrogénicos. Vet. Mex. 36: 437-452.

Pike, A.C.W., M. Brzozowski, A.E. Hubbard, R.T. Bonn, A. Thorsell, O. Engström, et al. 1999. Structure of the ligand-binding domain of oestrogen receptor beta in the presence of a partial agonist and a full antagonist. EMBO J. 18:4608-4618.

Romero, C., R. Tarrago, R. Muñoz, R. Arista, y A. Rosado. 1997. Síndrome estrogénico en vacas lecheras por consumo de alfalfas con grandes cantidades de coumestrol. Vet. Mex. 28:25-30.

Rosselli, M., K. Reinhart, B. Imthurn, P.J. Keller, and R.K. Dubey. 2000. Cellular and biochemical mechanisms by which environmental oestrogens influence reproductive function. Hum. Reprod. Update 6:332350.

Saunders, P.T.K. 2005. Does estrogen receptor $\beta$ play a significant role in human reproduction? Trends Endocrinol. Metabol. 16:222-227.

Sharma, V.D., R.F. Wilson, and L.E. Williams. 1994. Reproductive performance of female swine feed corn naturally molded or inoculated with Fusarium roseum Ohio isolates B and C. J. Anim. Sci. 38:598-602.

Shawn, I., and S. McCully. 2002. A review of the potential of dietary endocrine disrupters on the consumer. Int. J. Food Sci. Technol. 37:471-476.

Weihua, Z., S. Andersson, G. Cheng, E.R. Simpson, M. Warner, and J.A. Gustafsson. 2003. Update on estrogen signaling. FEBS Lett. 546:17-24.

Whitten P.L., and H.B. Patisaul. 2001. Cross-species and interassay comparisons of phytoestrogen action. Environ. Health Perspect. 109:5-20.

Whitten, P.L., H.B. Patisaul, and L.J. Young. 2002. Neurobehavioral actions of coumestrol and related isoflavonoids in rodents. Neurotoxicol. Teratol. 24:4754.

Yamada, T., A. Yamamoto, M. Fujioka, M. Miyagi, N. Saito, I. Imai, et al. 1999. Characterization of urinari metabolites of a new benzafuroquiniline derivative 3,9 - bis ( N, N-dimethylc arba moyloxy)- $5 \mathrm{H}$ benzofuro[3,2-C]-quinoline-6-one (KCA-098), in dogs. Pharmazie 54:672-677. 